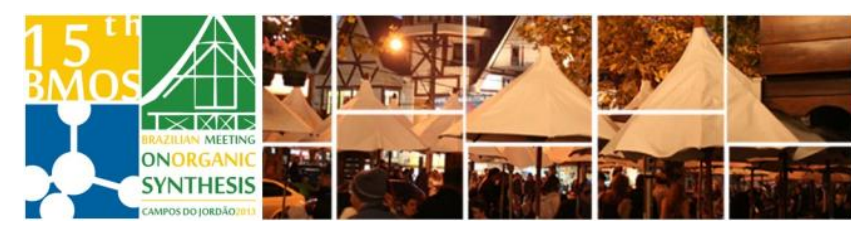

\title{
Phenanthrenequinone chlorination with trichloroisocyanuric acid
}

\section{Sirlene Oliveira Francisco de Azeredo and José Daniel Figueroa-Villar*}

Department of Chemistry, Military Institute of Engineering, Praça General Tiburcio, 80, 22290-270

Rio de Janeiro, RJ, Brazil. ("Figueroa@ime.eb.br)

Keywords: Phenanthrenequinone, trichloroisocyanuric acid and chlorination

\section{INTRODUCTION}

Halogenated aromatic compounds are used in the synthesis of products with important biological activity. Many methods of direct halogenation of aromatic systems involve the use of elemental halogen or expensive transition metal based catalysts. A reagent that has been used for chlorination of aromatic compounds is trichloroisocyanuric acid (TICA). ${ }^{1}$ TICA is a reagent which provides chlorination, being easy to access and manipulate, stable and inexpensive., ${ }^{2,3}$ Electrophilic aromatic substitution reactions with trichloroisocyanuric acid can be performed using polar aqueous solutions with sulfuric acid $50 \%$ (v/v) or a Lewis acid as catalyst. ${ }^{4}$ The objective of this work is to use acid trichloroisocyanuric for phenanthrenequinone chlorination using various methodologies to obtain chlorinated compounds employed as intermediates for the synthesis of new compounds with potential biological activity.

\section{RESULTS AND DISCUSSION}

The chlorinated compounds were synthesized reacting phenanthrenequinone (2 mmol) (1) and trichloroisocyanuric acid $(2 \mathrm{mmol})$ in diverse reactions conditions (Figure 1 ). After this time it was added $20 \mathrm{~mL}$ of distilled water and the precipitated product was filtered and washed with distilled water.

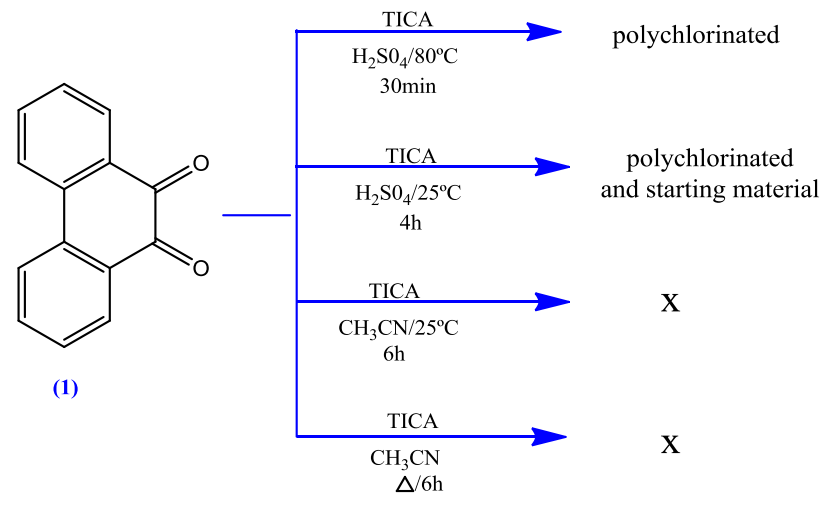

Figure1. Reactions conditions to tentative phenanthrenequinone (1) chlorination.
The more efficient method for phenanthrenequinone chlorination was using $\mathrm{H}_{2} \mathrm{SO}_{4}$ at $80^{\circ} \mathrm{C}$ for $30 \mathrm{~min}$. The products separation was initially tried by silica gel column chromatography using toluene as eluent. Later there were made silica preparative plates, using the same eluent.

It was possible to identify and characterize two polychlorinated compounds and the yields were of the $33 \%$ and $24 \%$ respectively to compounds 2 and 3. This separation procedure is still in progress.

The chemical shifts NMR ${ }^{1} \mathrm{H}$ and NMR ${ }^{13} \mathrm{C}$ of the compounds polychlorinated, which were obtained using gHSQC, gHMBC, gCOSY and APT are shown in Figure 2.
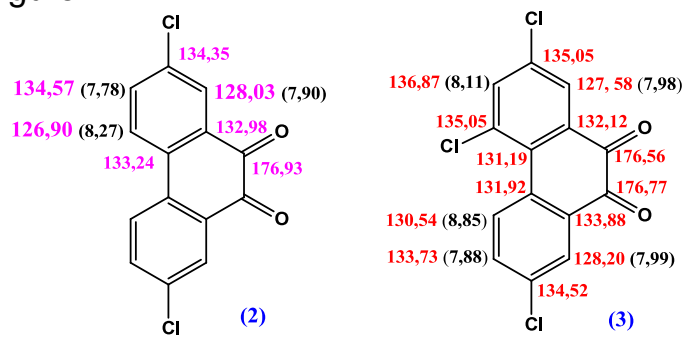

Figure 2: Chemical shifts of two polychlorinated compounds.

\section{CONCLUSION}

The results obtained were satisfactory and now are being characterized new derivatives that have been obtained for funcionalization of the phenanthrenequinone.

These products were prepared by an innovative procedure and are the intermediate materials for synthesis of new antibiotics and anticancer agents.

\section{ACKNOWLEDGEMENTS}

Acknowledgements to Ministery of Defence, CAPES, INBEB, FAPERJ and CNPq.

\section{REFERENCES}

${ }^{1}$ Mendonça, G.F et al. J. Chem. Soc., Vol.16 (4), p.695, 2005.

${ }^{2}$ Mendonça \& de Mattos. Química Nova, Vol. 31 (4), p.798, 2008.

${ }^{3}$ Vasconcellos \& Lima Junior. Química Nova, Vol. 32 (1), p.244, 2009

${ }^{4}$ Juenge, E.C; Beal, D.A; Duncan, W.P. J. Org. Chem. Vol. 35 (3), 719, 1970 